\title{
Impact of Frequency Offset on Interference between Zigbee and Wifi for Smart Grid Applications
}

\author{
Er. Anamika Vatsal ${ }^{1}$, Prof. Mehajabeen Fatima ${ }^{2}$ \\ Deptt.of Electronics \& Communication \\ Sagar Institute of Research \& Technology, Bhopal, India
}

\begin{abstract}
The Zigbee is a low cost communication technology used for low data rate communication system such as industrial automation etc. Because of its low complexity it is widely adopted for many applications. But the utilization of the same spectrum band by the WLAN system causes interference between both the systems. The proposed approach presents an analysis of this interference effect on Zigbee system when operated with WLAN sources at different distances and different power. It also analyzes the effect when a frequency offset is established between both systems. The simulation results shows that a small offset can provide sufficient improvement in the performance.
\end{abstract}

Keywords: Smart Grids, Zigbee Network, Mesh Network, Wireless LAN (WLAN), BER.

\section{Introduction}

The application of the electronics is gaining importance in the industrial automations because of its better performance and lower possibility of error and low cost as compared with manual controlling. A smart grid is a modernized electrical grid that uses information and communications technology to gather and act on information, such as information about the behaviors of suppliers and consumers, in an automated fashion to improve the efficiency, reliability, economics, and sustainability of the production and distribution of electricity. Although to effectively operate such systems it is required to gather the information from the various parts of the plant and then it is processed by a central processing system. Since it is difficult and costly to take wires through every part of plant, an effective wireless solution is required. It is also noted that in most of the cases, only small amount of data is required to send. All these requirements make the zigbee as an optimal solution because it provides low cost, large range, low power, and low complexity solution at $250 \mathrm{Kbits} / \mathrm{s}$ data rate along with supporting various topologies. Studies have shown that till now the Zigbee is successfully applied for industrial automation, wireless sensor network, home automation, building automation and medical data collection, smart grids etc. Because of many such applications, it is required to investigate the performance of zigbee under different physical scenarios to make it an effective and robust communication system which can be used for critical applications such as life supporting system. This paper is also an effort towards it. The numerous challenges underlying due to interference amongst Zigbee and WiFi in the $2.4 \mathrm{GHz}$ ISM band served as the motivation for this work. Despite of the extensive existing research in Zigbee and WiFi coexistence, no work regarding the influence of comparative study of frequency offset on BER and SINR using matlab software can be found in the related work. The rest of the paper is organized in the following manner. The second section provides a brief review of the related works. The third section describes the operation of Zigbee followed by the working of WLAN in fourth section. The fifth section presents the proposed work and the sixth section details the simulation results for the proposed work. Finally, the conclusion is drawn in section seventh section.

\section{Literature Review}

This section presents a brief review of the literatures related to the same field. Wail Mardini et al [1] studied the mutual impact of WiFi interference on ZigBee channels by using QualNet Simulator and computed the Packet Delivery Rate (PDR) for both ZigBee and WiFi nodes. The obtained results show that ZigBee has little impact on WiFi Packets, and WiFi affects ZigBee packets with different degrees based on the traffic intensity and number of nodes. James Hou et al [2] proposed the interference minimizing technique between 802.11 and ZigBee medical sensors. Chieh-Jan Mike Liang et al [3] designed BuzzBuzz to mitigate WiFi interference through header and payload redundancy. Multi-Headers provides header redundancy giving ZigBee nodes multiple opportunities to detect incoming packets. Then, TinyRS, a full-featured Reed Solomon library for resource-constrained devices, helps decoding polluted packet payload. On a medium-sized testbed, BuzzBuzz improves the ZigBee network delivery rate by $70 \%$. Furthermore, BuzzBuzz reduces ZigBee retransmissions by a factor of three, which increases the WiFi throughput by $10 \%$. Jun Huang et al [4] proposed a novel approach that enables ZigBee links to achieve assured performance in the presence of heavy WiFi interference. First, based on statistical analysis of real-life network traces, they present a Pareto model to accurately characterize the white space in WiFi traffic. Second, we analytically model the performance of a 
ZigBee link in the presence of WiFi interference. Third, based on the white space model and our analysis, we develop a new ZigBee frame control protocol called WISE, which can achieve desired trade-offs between link throughput and delivery ratio. Their extensive experiments on a testbed of 802.11 netbooks and 802.15.4 TelosB motes show that, in the presence of heavy WiFi interference, WISE achieves $4 \mathrm{x}$ and $2 \mathrm{x}$ performance gains over B-MAC and a recent reliable transmission protocol, respectively, while only incurring $10.9 \%$ and $39.5 \%$ of their overhead. Yanchao Mao et al [5] presented an experimental analysis of the interference between IEEE 802.15.4 and IEEE $802.11 \mathrm{~b} / \mathrm{g}$ and their measurement results are valuable for the real world deployment and design of the network integrated IEEE 802.15.4 and IEEE $802.11 \mathrm{~b} / \mathrm{g}$.

\section{Zigbee Communication}

ZigBee is a specification for a suite of high level communication protocols used to create personal area networks built from small, low-power digital radios. ZigBee is based on an IEEE 802.15 standard. Though lowpowered, ZigBee devices often transmit data over longer distances by passing data through intermediate devices to reach more distant ones, creating a mesh network; i.e., a network with no centralized control or high-power transmitter/receiver able to reach all of the networked devices. The decentralized nature of such wireless ad hoc networks makes them suitable for applications where a central node can't be relied upon. ZigBee is used in applications that require a low data rate, long battery life, and secure networking. ZigBee has a defined rate of $250 \mathrm{Kbit} / \mathrm{s}$, best suited for periodic or intermittent data or a single signal transmission from a sensor or input device. Applications include wireless light switches, electrical meters with in-home-displays, traffic management systems, and other consumer and industrial equipment that requires short-range wireless transfer of data at relatively low rates. The technology defined by the ZigBee specification is intended to be simpler and less expensive than other WPANs, such as Bluetooth or Wi-Fi. ZigBee networks are secured by 128 bit symmetric encryption keys. In home automation applications, transmission distances range from 10 to 100 meters line-of-sight, depending on power output and environmental characteristics [1]. ZigBee was conceived in 1998, standardized in 2003 and revised in 2006. The name refers to the waggle dance of honey bees after their return to the beehive [2]. ZigBee operates in the industrial, scientific and medical (ISM) radio bands; $868 \mathrm{MHz}$ in Europe, $915 \mathrm{MHz}$ in the USA and Australia and $2.4 \mathrm{GHz}$ in most jurisdictions worldwide. Data transmission rates vary from 20 kilobits/second in the $868 \mathrm{MHz}$ frequency band to 250 kilobits/second in the $2.4 \mathrm{GHz}$ frequency band [3].

The ZigBee network layer natively supports both star and tree typical networks, and generic mesh networks. Every network must have one coordinator device, tasked with its creation, the control of its parameters and basic maintenance. Within star networks, the coordinator must be the central node. Both trees and meshes allows the use of ZigBee routers to extend communication at the network level.

\section{Wireless Lan (Wlan), Ieee 802.11}

A wireless local area network (WLAN) links two or more devices using some wireless distribution method (typically spread-spectrum or OFDM radio), and usually providing a connection through an access point to the wider Internet. This gives users the mobility to move around within a local coverage area and still be connected to the network. Most modern WLANs are based on IEEE 802.11 standards, marketed under the Wi-Fi brand name.

IEEE 802.11 is a set of medium access control (MAC) and physical layer (PHY) specifications for implementing wireless local area network (WLAN) computer communication in the 2.4, 3.6, 5 and $60 \mathrm{GHz}$ frequency bands. They are created and maintained by the IEEE LAN/MAN Standards Committee (IEEE 802). The base version of the standard was released in 1997 and has had subsequent amendments. The standard and amendments provide the basis for wireless network products using the Wi-Fi brand. While each amendment is officially revoked when it is incorporated in the latest version of the standard, the corporate world tends to market to the revisions because they concisely denote capabilities of their products. As a result, in the market place, each revision tends to become its own standard.

The 802.11 family consist of a series of half-duplex over-the-air modulation techniques that use the same basic protocol. The most popular are those defined by the $802.11 \mathrm{~b}$ and $802.11 \mathrm{~g}$ protocols, which are amendments to the original standard. 802.11-1997 was the first wireless networking standard in the family, but 802.11a was the first widely accepted one, followed by $802.11 \mathrm{~b}$ and $802.11 \mathrm{~g}$. $802.11 \mathrm{n}$ is a new multi-streaming modulation technique. Other standards in the family $(\mathrm{c}-\mathrm{f}, \mathrm{h}, \mathrm{j})$ are service amendments and extensions or corrections to the previous specifications.

$802.11 \mathrm{~b}$ and $802.11 \mathrm{~g}$ use the $2.4 \mathrm{GHz}$ ISM band, operating in the United States under Part 15 of the U.S. Federal Communications Commission Rules and Regulations. Because of this choice of frequency band, $802.11 \mathrm{~b}$ and g equipment may occasionally suffer interference from microwave ovens, cordless telephones and Bluetooth devices. $802.11 \mathrm{~b}$ and $802.11 \mathrm{~g}$ control their interference and susceptibility to interference by using 
direct-sequence spread spectrum (DSSS) and orthogonal frequency-division multiplexing (OFDM) signaling methods, respectively.

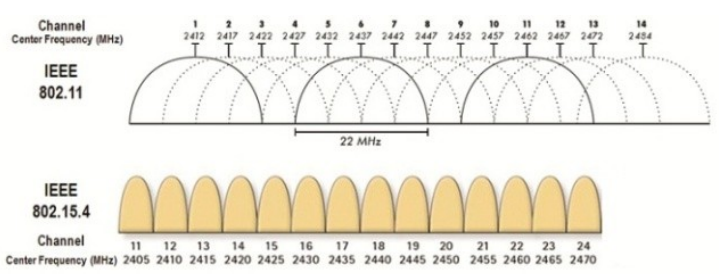

Figure 1: Zigbee and WLAN channels in $2.4 \mathrm{GHz}$ band[12]

802.11a uses the $5 \mathrm{GHz}$ U-NII band, which, for much of the world, offers at least 23 non-overlapping channels rather than the $2.4 \mathrm{GHz}$ ISM frequency band, where adjacent channels overlap and contain only 3 nonoverlapping channels as shown in Fig. 1. Better or worse performance with higher or lower frequencies (channels) may be realized, depending on the environment.

The segment of the radio frequency spectrum used by 802.11 varies between the countries. In the US, 802.11a and 802.11g devices may be operated without a license, as allowed in Part 15 of the FCC Rules and Regulations. Frequencies used by channels one through six of $802.11 \mathrm{~b}$ and $802.11 \mathrm{~g}$ fall within the $2.4 \mathrm{GHz}$ amateur radio band. Licensed amateur radio operators may operate $802.11 \mathrm{~b} / \mathrm{g}$ devices under Part 97 of the FCC Rules and Regulations, allowing increased power output but not commercial content or encryption.

\section{Proposed Work}

In this paper, we presented an analysis of interference between IEEE 802.11 systems and Zigbee operating under $2.4 \mathrm{GHz}$ band. The simulation is made using Matlab v 7.5 software. In the simulation, it is assumed that both the systems are continuously transmitting and receiving data at a fixed rate while the distance and the power of the WiFi transmitter are being be varied as per the analysis requirements.

The channel is considered to be a Rayleigh channel assuming no dominant propagation along the line of sight. Additive White Gaussian Noise(AWGN) is introduced within this channel.

The zigbee model is developed in accordance with IEEE 802.15.4 standards document, every four bits are mapped into a symbol and each symbol spreads to a 32-chip almost orthogonal PN sequence, thus a spreading table is set in a spreading block. The transmission rate is $250 \mathrm{kbps}$ at $2.4 \mathrm{GHz}$ for ZigBee, while 11 Mbps for WiFi.

\subsection{Measures used for Analysis}

BER:The bit error rate or bit error ratio (BER) is the number of bit errors divided by the total number of transferred bits during a studied time interval[14]

$$
B E R=\frac{\text { Total Errors }}{\text { Total Bits }}
$$

SINR:signal-to-interference-plus-noise ratio (SINR) which can be defined as [13]

$$
\operatorname{SINR}=\frac{P_{\text {signal }}}{P_{\text {noise }}+P_{\text {interference }}}
$$

Path Loss: The path loss model represents the power loss between transmitter and receiver and is given by [15]

$$
L_{p}(d)=\left\{\begin{array}{c}
20 \log _{10}\left(\frac{4 \pi d}{\lambda}\right), d \leq d_{0} \text { else } \\
20 \log _{10}\left(\frac{4 \pi d}{\lambda}\right)+10 n \log _{10}\left(\frac{d}{d_{0}}\right)
\end{array}\right\}
$$

Where $d_{0}$ is a break point. We set $n$ equals to 3.3 and $d_{0}$ is $8 \mathrm{~m}$.

\section{Scenario 1:}

\section{Simulation Results}

In this scenario, the signal to interference ratio is estimated for different distances between zigbee receiver and the Wifi transmitter. Here the zigbee transmitter is placed at 1 meter distance of its receiver while the distance of Wifi transmitter is increased gradually from 1 meter to 10 meters and the value of AWGN is $3 \mathrm{~dB}$ and the Wifi is working at 11 mbps data rate. 


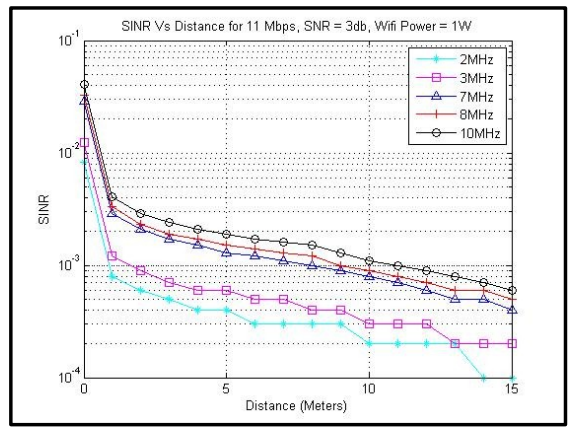

Figure 2a: Simulated SINR performances for different offset frequencies with varying distance, the graph shows that offset improves the SINR by 10 times when offset increased from 2 to $10 \mathrm{Mhz}$ for every value of distance.

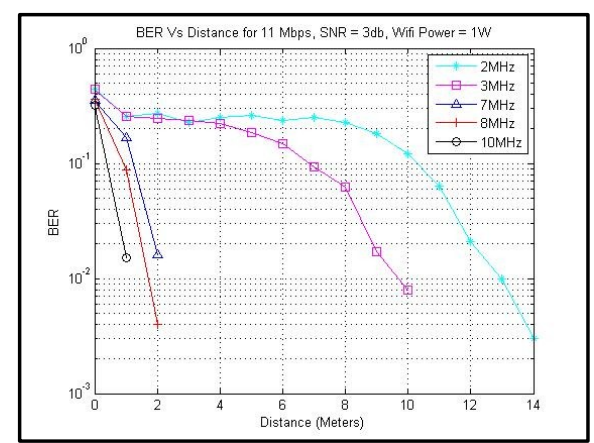

Figure 2b: Simulated BER performances for different offset frequencies with distance variation. The graph shows that the BER increases exponentially with offset frequency increment.

\section{Scenario 2:}

All parameters are similar to first scenario except the AWGN varies and the distance is remain constant to 1 meter.

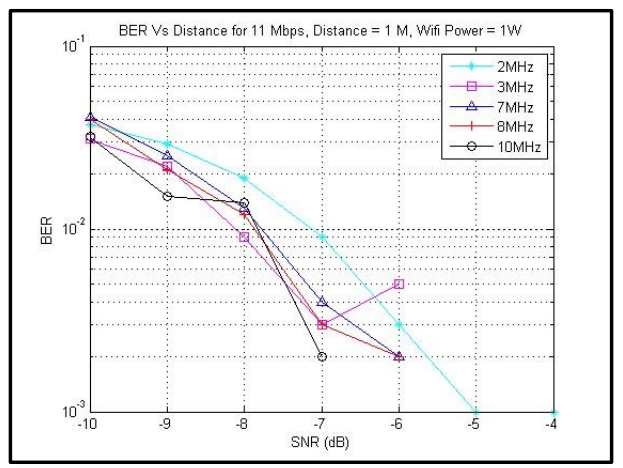

Figure 3: Simulated BER performances for different offset frequencies with varying AWGN.The offset variation doesn't affect the BER variation (overlapping graphs). The variation in BER is due to SINR only.

\section{Scenario 3:}

In this scenario, the signal to interference ratio is estimated for different distances between zigbee receiver and the Wifi transmitter. Where the power of zigbee transmitter is placed at 1 meter distance of its receiver while the distance of Wifi transmitter is increased gradually from 1 meter to 10 meters and the value of AWGN is $-3 \mathrm{~dB}$ and the Wifi is working at $5.5 \mathrm{mbps}$ data rate. 


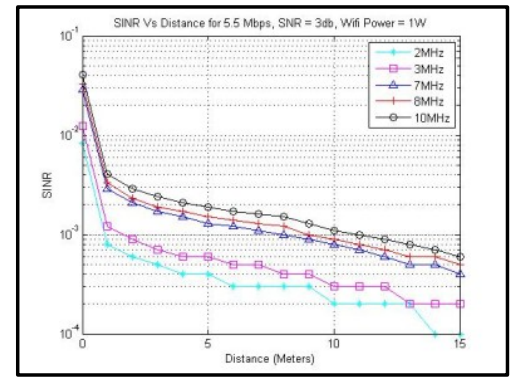

Figure 4a: Simulated BER performances for different offset frequencies with distance variation. The graph shows that the BER decreases exponentially with offset frequency increment.

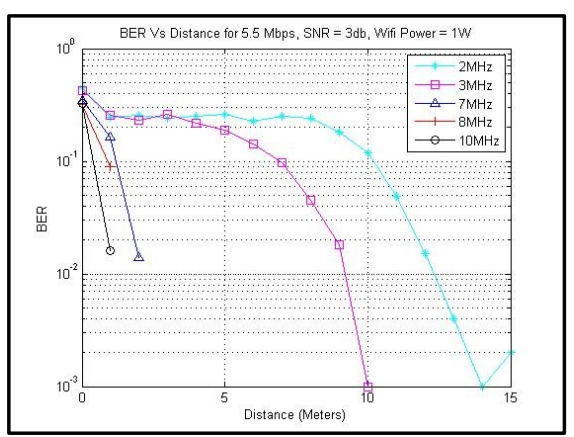

Figure 4b: Simulated BER performances for different offset frequencies with distance variation. The graph shows that the BER decreases exponentially with offset frequency increment.

\section{Scenario 4:}

All parameters are similar to first scenario except the AWGN varies and the distance remains constant to 1 meter.

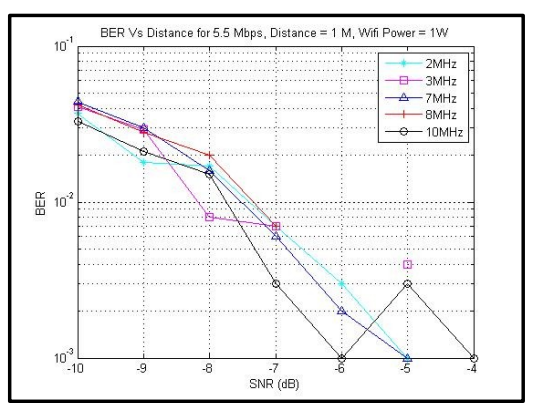

Figure 5: Simulated BER performances for different offset frequencies with varying AWGN. The offset variation doesn't affect the BER variation(overlapping graphs).The variation in BER is due to SINR only.

\section{Conclusion}

In the paper, the simulation for the analysis of the interference is performed for different conditions and the values of SINR and the BER is measured. From the results it can be concluded that the interference between these systems can be avoided by providing the small offset which can reduce the SINR by 10 times (refer to graphs 1a and 3a) and BER due to interference by 100 times (refer to graphs $1 \mathrm{~b}$ and 3b). BER due to interference is computed and analyzed for frequencies above $8 \mathrm{MHz}$ which is considerably beneficial towards the smart grid applications and is true for both the data rates. The offset variation, however, doesn't improve the AWGN performance of the system(refer to graphs 3 and 5). Hence we can say that small frequency offset can be used instead of incorporating complex protocols. Nevertheless, this paper talks about the analysis and observation of offset frequency to trace out the behavior of BER and SINR. This work can further be carried out for other wireless technologies like WiMax with comparatively lower interference than Zigbee.

\section{Acknowledgement}

The Author would like to thanks The Rajiv Gandhi Proudyogiki Vishwavidyalaya, Bhopal for its generous support and the Sagar Institute of Research \& Technology, Bhopal for their hospitality during my academic period 2011-2013. She wishes to thanks Prof. Mehajabeen Fatima for her help and encouragement. 


\section{References:}

[1] Howitt, I, Gutierrez, JA. ZigBee low rate-wireless personal area network coexistence issues. Wireless Communications and Networking, 2003; (3): 1481- 1486.

[2] Axel S, Voicu FG. Coexistence of ZigBee with other Systems in the 2.4 GHz-ISM-Band. In IEEE Instrumentation and Measurement Technology' 2005 May, Ottawa: Canada.1786-1791.

[3] Marina P, Janne R, Petri M, Saverio L. Performance Study of ZigBee Using Measurements and Simulations. Proceedings of IEEE Wireless Communications and Networking Conference 2006 April ; Las Vegas, NV:USA; 487-492.

[4] Soo YS, Hong SP, Wook HK. Mutual interference analysis of ZigBee and WiFi-b. Computer and Telecommunications Networking 2007;(51) : 3338-3353.

[5] HUO H, XU Y, Mikael G, ZHANG H. Coexistence of $2.4 \mathrm{GHz}$ sensor networks in home environment. China Universities of Posts and Telecommunications 2010; 17(1):9-18.

[6] Antoine BB, Ashish M, Marco Z. Experimental Evaluation of Interference Mitigation on The 2.4 GHz ISM band Using Channel Hopping, Proceedings of the IEEE Wireless Days' October 2010; Venice:Italy: 1-5.

[7] Jun H, Guoliang X, Gang Z, Ruogu Z. Beyond Co-existence: Exploiting WiFi White Space for ZigBee Performance Assurance. The 18th IEEE International Conference on Network Protocols (ICNP)' October 2010; Kyoto: Japan. 305-314.

[8] Shahin F. ZigBee Wireless Networks and Transceivers. USA: Newnes; 2008: 339. ISBN: 978-0-7506-8393-7.

[9] Cirronet, Inc. ZigBee ${ }^{\mathrm{TM}}$ Wireless Transceiver Engineering Options. 2005. White Paper.

[10] Paolo B, Prashant P, Vince WC Chook, Stefano C, Alberto G, YFun Hu. Wireless Sensor Networks: A Survey on the State of the Art and the 802.15.4 and ZigBee Standards. Computer Communications. 2007; (30):1655-1695.

[11] William L, Lee W McKnight. Wireless Internet Access: 3G vs. WiFi Madrid. Telecommunications Policy 2003. 351-370.

[12] www.ni.com: $2.4 \mathrm{GHz} \_$Spectrum.jpg

[13] Peizhong Yi, Abiodun Iwayemi and Chi Zhou. "Developing ZigBee Deployment Guideline Under WiFi Interference for Smart Grid Applications" in IEEE Transactions on Smart Grids, 2011 March, Chicago, USA : 1949-3053.

[14] Ron Franac. "BER and MER Fundamentals" by CISCO Systems, 2007.

[15] Soo Young Shin, Jeong Seok Kang, Hong Seong Park. "Packet Error Rate Analysis of ZigBee under Interferences of Multiple Bluetooth Piconets" in IEEE, April, 2009; ISBN: 978-1-4244-2517 